\title{
Drahtlose energieautarke RFID-Sensorik im Motorraum von Kraftfahrzeugen
}

\author{
Jakob Happel, Johann Harmstorf, Karl-Ludwig Krieger \\ ITEM Universität Bremen, Otto-Hahn-Allee 1, 28359 Bremen, Germany
}

\section{Zusammenfassung}

Heutige verbaute konventionelle Sensoren in Kraftfahrzeug haben eine drahtgebundene externe Energieversorgung sowie Signalleitungen zur Kommunikation. Für die Entwicklung der nächsten Generation werden kostendämpfende Maßnahmen bestimmend sein, welche entweder durch Produktund Produktionsoptimierung oder durch den Einsatz neuartiger autarker Sensorsysteme erzielt werden können. In diesem Beitrag wird ein innovatives Konzept zur Integration drahtloser energieautarker Sensorknoten im Motorraum vorgestellt, bei dem die Energie- und Datenübertragung mithilfe der RFID-Technologie realisiert wird.

Als RFID-Basisstation dient ein zentrales Steuergerät mit einer HF-geeigneten Antennenstruktur, welche beispielsweise im Dämmmaterial der Motorhaube integriert ist. Diese Basisstation ermöglicht eine Erfassung von Messdaten unterschiedlicher Sensorknoten im gesamten Motorraum und übermittelt die gewonnen Informationen an einen fahrzeuginternen Kommunikationsbus.

Das Hauptziel der Entwicklung stellen autarke Sensorsysteme zur aktiven Erfassung von Messgrößen mit geringen Latenzzeitanforderungen, wie beispielsweise Füllständen, Temperaturen etc., dar. Neben den aktiven Sensorsystemen können die Sensorknoten auch ohne aktive Messaufgaben rein passiv als RFID-Etiketten ausgelegt sein, z.B. zur Erkennung von inkompatiblen Austauschteilen.

Keywords: RFID, Energieautarke Sensoren, Füllstandserfassung, Energy Harvesting

\section{Motivation}

Die Integration sensorbasierter Funktionen in das Kraftfahrzeug steigt stetig an. Es werden immer mehr Sensoren zur Realisierung von Basis- und Komfortfunktionen im Fahrzeug eingesetzt. Diese bilden die Grundlage zahlreicher Sicherheitssysteme, ermöglichen einen effizienten Betrieb des Fahrzeugs und erleichtern die Bedienung durch den Fahrzeugführer. Weiterhin werden die Fehlerdiagnose und auch die Wartung des Fahrzeugs durch sensorbasierte Systeme vereinfacht.

Ein Innovationshindernis für die Integration sensorbasierter Funktionen ins Fahrzeug stellt die Verkabelungs- und Verbindungstechnik dar, deren Umfang sich durch digitale, serielle Bussysteme z. B. CAN-Bus reduzieren, jedoch nicht vollständig vermeiden lässt. Heutige verbaute konventionelle Sensoren im Kraftfahrzeug benötigen eine drahtgebundene externe Energieversorgung sowie zusätzliche Signalleitungen zur Kommunikation. In modernen PKW der Oberklasse beträgt die Leitungslänge trotz Vernetzung bis zu $3 \mathrm{~km}$ [1] mit einem Gewicht von bis zu $30 \mathrm{~kg}$. Die verbauten Kabel erhöhen das Gesamtgewicht und beeinflussen damit den Kraftstoffverbrauch. Weiterhin stellt die Verkabelung erhebliche Anforderungen an den
Bauraum innerhalb des Fahrzeugs und der betroffenen Komponenten. Neben den installierten Kosten sind Kabel und Steckverbinder potentielle Schwachstellen. Auftretende Störungen werden häufig durch unterbrochene Verbindungen zwischen Steuergeräten und Sensoren verursacht. Durch den hohen Personalaufwand bei Installation und Wartung ist die Verkabelung im Kraftfahrzeug mit hohen Kosten verbunden.

Für die Entwicklung der nächsten Generation werden kostendämpfende Maßnahmen bestimmend sein, welche entweder durch Produkt- und Produktionsoptimierung oder durch den Einsatz neuartiger autarker Sensorsysteme erzielt werden. Eine Möglichkeit den Verkabelungsaufwand zu minimieren und dabei dennoch die Anzahl der Sensoren zu erweitern ist die Integration energieautarker drahtloser sensorbasierter Mikrosysteme direkt in die zu überwachenden Komponenten. Durch diese Maßnahme können Installationskosten, Bauraum, Gewicht und vor allem Kabelverbindungen zur Energieversorgung und Kommunikation der Sensorsysteme eingespart werden. 


\section{Stand der Technik: Energieautarke Senso- rik im Kraftfahrzeug}

Die Idee eines energieautarken Sensorknotens zur Kostenreduktion von Installations- und Wartungskosten im Automobil ist Gegenstand mehrerer Forschungsprojekte und Veröffentlichungen. Die Leistungsfähigkeit autarker drahtloser Sensorsysteme hängt dabei entscheidend von ihrer Energieversorgung und ihrem Energiemanagement ab. Ein durch erschöpfliche Energiespeicher wie Batterien und Akkus betriebenes System ist wartungsbedürftig und damit nur bedingt für den Einsatz im Fahrzeug geeignet. Die Energieernte aus der direkten lokalen Umgebung hingegen stellt ein innovatives, aussichtsreiches Konzept zur wartungsfreien Energieversorgung von Sensorsystemen dar. Mit Hilfe unterschiedlicher Wandlungsprinzipien kann Energie aus thermischen, kinetischen oder elektromagnetischen Quellen mit geeigneten Generatoren in elektrische nutzbare Energie umgewandelt werden. Die Energieerzeugung aus Vibrationen ist derzeit auf Grund der speziellen Umgebung des Motorraums die vielversprechendste und einzig universelle Möglichkeit der Energieernte für Mikrosysteme, da diese im gesamten Motorraum vorliegen. Der Ansatz der Energieernte für Mikrosysteme wird in einigen Anwendungen im KFZ-Bereich verfolgt [2,3,4]. Die nutzbaren Energiepotentiale im Motorraum, die durch Vibrationen verursacht werden, waren bereits Gegenstand einer eigenen Untersuchung [5]. Als Ergebnis konnte festgehalten werden, dass ein rein aus den Motorvibrationen versorgtes Sensorsystem prinzipiell zwar umsetzbar, die Leistungsfähigkeit dieser Systeme allerdings auf Grund von energetischen Restriktionen stark eingeschränkt ist. Zusätzlich ist eine gezielte Adaption an das jeweilige Fahrzeug, den Einbauort und z.T. auch an das personenspezifische Fahrverhalten notwendig, sodass ein universeller Einsatz derzeit nicht sinnvoll erscheint ist. Die Entwicklung eines solchen Systems ist daher in der Umsetzung sehr komplex und nur für Spezialanwendungen mit hoher Applikationsadaption empfehlenswert. Aus diesem Grund wird innerhalb dieser Veröffentlichung ein geeigneteres, universelleres Konzept zur Integration drahtloser energieautarker Sensorsysteme im Motorraum verfolgt.

\section{Stand der Technik: Füllstandserfassung im Kraftfahrzeug}

Im Bereich des Kraftfahrzeugs werden Treibstoff-, Kühlwasser-, Öl- oder Bremsflüssigkeitsbehälter mithilfe elektronischer Sensorsysteme überwacht. Aktuelle Messsysteme zur Füllstandsdetektion im Kraftfahrzeug bestehen hauptsächlich aus elektromechanischen Systemen. Bei der kontinuierlichen Füllstandserfassung im Kraftstofftank wird ein Schwimmer mit geringer Dichte, dessen Position durch das Niveau des Fluides verändert wird, in dem zu überwachenden Behälter platziert. Der Schwimmer ist über einen metallischen Hebel mechanisch mit einem resistivem Wegaufnehmer verbunden, dessen Widerstand sich je nach Füllstand ändert und durch eine Analog-Digital-Wandlung eines Mikrocontrollers ausgewertet werden kann.

Die Überwachung des Flüssigkeitsfüllstands in den Behältern eines Kraftfahrzeugs wie Scheibenwischwasser, Kühlflüssigkeit, Öl für Motor, Getriebe und Servolenkung und Bremsflüssigkeit wird derzeit nur als Warnhinweis bei minimalem Niveau realisiert. Technisch wird diese Funktion durch einen digitalen Schwellwertschalter umgesetzt, wobei eine kontinuierliche Erfassung des Füllstands derzeit nicht durchgeführt wird. Der Mehrwert einer kontinuierlichen Erfassung wäre zunächst eine Steigerung des Bedienkomforts, allerdings kann das frühzeitige zustandsorientierte Auffüllen des jeweiligen Behälters das Entstehen von sicherheitsgefährdenden Situationen durch unzureichende Befüllung verhindern.

Konventionelle mechanische Messsysteme mit beweglichen Komponenten sind anfällig für Verschleiß und Korrosion. Die metallischen Kontakte der resisitiven Wegaufnehmer nutzen sich im Betrieb durch die mechanische Belastung ab. Dadurch wird die Messgenauigkeit verringert und das Messsystem bei übermäßiger Belastung beschädigt. Auf Grund der genannten Nachteile des mechanischen Messprinzips wurde dieses Konzept für das angestrebte Sensorsystem verworfen und stattdessen eine kontaktlose Messmethode ohne bewegliche Komponenten angestrebt, da diese prinzipiell verschleißfrei ist und dadurch eine höhere Langzeitstabilität aufweist.

\section{Darstellung des Systemkonzepts}

Das in dieser Veröffentlichung umgesetzte Konzept sieht eine gezielte elektromagnetische Einstrahlung der für die sensorischen Funktionen notwendigen Energie an lokal verteilte, vollständig autarke Sensorknoten vor. Als Basisstation zur Energie- und Datenübertragung dient ein zentrales Steuergerät mit einer geeigneten Antennenstruktur, welche beispielsweise im Dämmmaterial der Motorhaube installiert ist. Abbildung 1 stellt das energieautarke Sensorkonzept im Motorraum dar, wobei die Anzahl der Sensorknoten beliebig erweiterbar ist. 


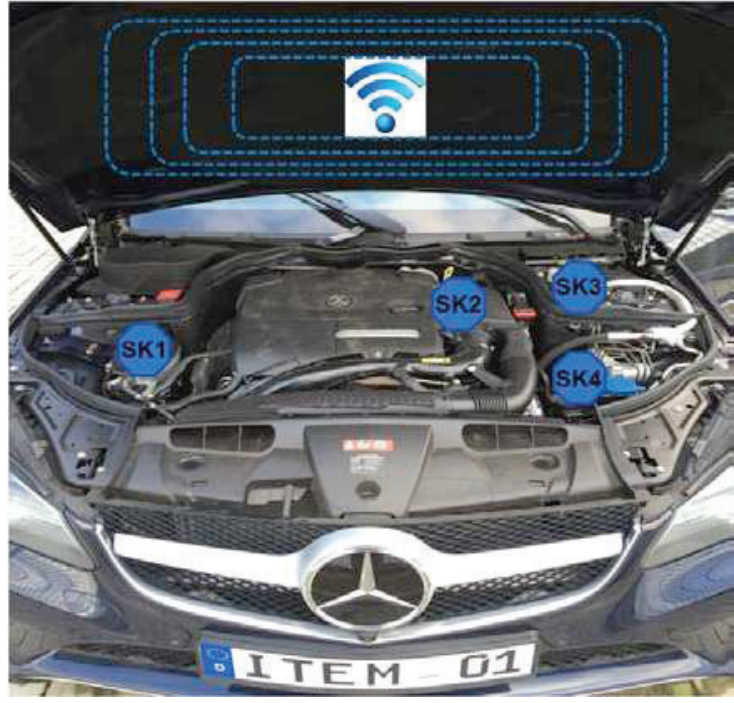

\section{Abb. 1: Systemdarstellung}

Die Basisstation ermöglicht eine Erfassung von Messdaten unterschiedlicher Sensorknoten im gesamten Motorraum und übermittelt die gewonnen Informationen an einen fahrzeuginternen Kommunikationsbus. Die Sensorknoten können zum einen ohne aktive Messaufgaben rein passiv als RFID-Etiketten ausgelegt sein, z.B. zur Erkennung von inkompatiblen Austauschteilen. Neben der passiven Identifikation, können auch aktive autarke Sensorknoten, im Folgenden als Sensorsysteme bezeichnet, zur Erfassung von Messgrößen mit geringen Latenzzeitanforderungen wie beispielsweise Füllständen, Temperaturen etc. ausgelesen werden.

Die Basisstation ermöglicht ein zentrales Auslesen aller Sensordaten mit einer RFIDAntenne. Eine zentrale Steuereinheit realisiert die Ablaufsteuerung der Datenerfassung und wertet die Messdaten der Sensorsteuereinheit aus. Anschließend werden die Messdaten mithilfe eines Moduls in einen Fahrzeugbus wie z.B. CAN übertragen. Der Signalfluss der Sensordaten ist in Abbildung 2 dargestellt.

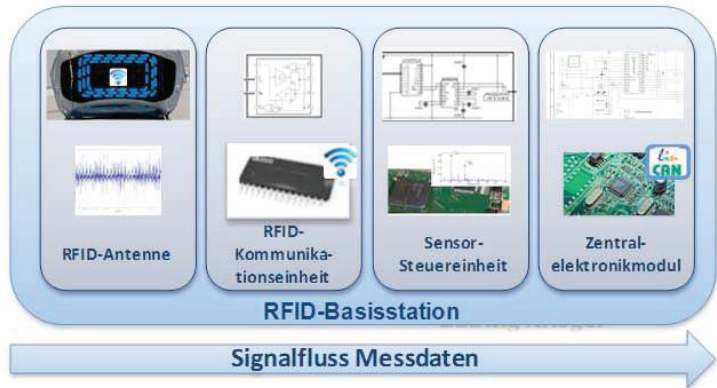

\section{Abb. 2: Signalfluss der Messdaten innerhalb der Basisstation}

Der prinzipielle Aufbau eines aktiven Sensorknotens ist in Abbildung 3 zu sehen. Ein geeignetes analoges Front-End übernimmt die drahtlose Energie- und Datenübertragung seitens des Sensorknotens. Der zentrale Bestandteil ist ein Low-Power-Mikrocontroller zur Ablaufsteuerung. Dieser ermöglicht eine digitale und analoge Anbindung von diversen externen Sensoren zur Bestimmung von bspw.

- Füllstand

- Medienkonzentration

- Temperatur

- Druck

- etc.

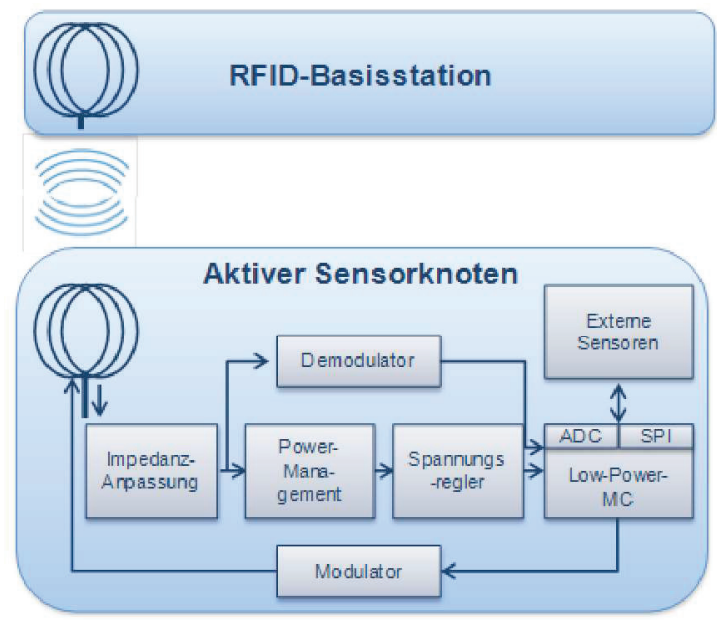

\section{Abb. 3: Darstellung aktives Sensorsystem}

Eine zentrale Herausforderung bei funkbasierten Systemen mit drahtlosem Datenaustausch stellen häufig der Datenschutz und die Störanfälligkeit dar. Im Bereich des Datenschutzes hat die RFID-Technologie einen grundsätzlichen Vorteil gegenüber konventionellen funkbasierten Systemen mit eigener Energieversorgung, da diese systembedingt nur eine eingeschränkte Reichweite über wenige Meter ermöglicht. Zudem verringert die Verwendung einer gerichteten Antenne im Motorraum den Radius, in dem eine unbefugte Manipulation der übertragenen Kommunikationsdaten stattfinden kann, z.B. durch Abhören oder auch Einspeisen von Daten ins System. Desweiteren fungieren die Motorhaube und andere metallische Karosseriekomponenten als EMVSchirmung gegenüber dem Außenbereich des Motorraumes und stellen dadurch eine physikalische Sicherung der Kommunikation dar.

Eine Herausforderung für eine zuverlässige drahtlose Kommunikation und die Störsicherheit stellen diverse metallische Oberflächen und teilweise auch die Flüssigkeiten selbst dar. Eine metallische Komponente zwischen der Basisantenne und dem Transponder verursacht eine Schirmung der ausgestrahlten elektromagnetischen Signale und führt dazu, dass die Signalstärke am Transponder stark einbricht oder diese z.T. vollständig ausfallen. In diesem Zusammenhang ist eine generelle 
Aussage zur Verfügbarkeit der Transponder im Motorraum nicht möglich, da die spezifische Einbaukonstellation des jeweiligen Fahrzeugs berücksichtigt werden muss. Allerdings werden Flüssigkeitsbehälter und Verschleißteile wie z.B. Filter im Motorraum größtenteils nach oben ausgerichtet verbaut, um eine einfache Wartung bzw. den Austausch zu ermöglichen. Dementsprechend wird die Problematik des metallischen Systemumfelds für die Realisierung der angestrebten sensorischen Funktionen im Sinne des Systemkonzepts entschärft. In dieser Veröffentlichung liegt der Fokus insbesondere auf energieautarken Sensorknoten zur Füllstandsdetektion. Zur Minimierung der installierten Kosten ist dieses System preiswert und universell einsetzbar auszulegen, sodass das Sensorsystem in unterschiedliche Flüssigkeitsbehälter des Motorraums mit geringen Anpassungen integriert werden kann. Dabei soll der Messwertaufnehmer einen deutlich geringeren Bauraum gegenüber mechanischen Sensorsystemen beanspruchen und dadurch in hohem $\mathrm{Maße}$ in die Flüssigkeitsbehälter integrierbar sein.

In den folgenden Abschnitten wird auf die Möglichkeiten zur Realisierung unterschiedlicher Sensorknoten mit verschiedenen Messfunktionen eingegangen.

Dabei werden zunächst RFID-Transponder in der Ausführung als preiswerte Klebeetiketten zur gezielten Wartung von Verschleißkomponenten vorgestellt. Diese gehören schon heute zu einer etablierten Technologie und werden deshalb nur kurz dargestellt.

Im anschließenden Kapitel wird ein passives Verfahren zur Auswertung des Füllstands anhand der Signaldämpfung beschrieben. Hierbei werden einleitend die für das Verständnis notwendigen theoretischen Grundlagen hergeleitet und anschließend eine messtechnische Verifikation anhand eines Demonstrationssystems durchgeführt.

Der vorletzte Abschnitt behandelt aktive RFIDSensorsysteme, bei denen die elektronischen Komponenten zur aktiven Messdatenerfassung aufgrund ihres hohen Energiebedarfs nicht ausreichend durch den RFID-Übertragungskanal versorgt werden können. Diese benötigen ein Sekundärsystem zur Energieversorgung, dessen Realisierung in diesem Kapitel beschrieben wird.

\section{RFID-Etiketten zur gezielten Wartung von Verschleißkomponenten}

Der Wartungszeitpunkt eines Fahrzeugs wird bereits heutzutage durch einen Warnhinweis des Bordcomputers an den Fahrer weitergegeben. Allerdings wird beim Service zwischen unterschiedlichen Servicearten unterschieden. Welche Komponenten bei der Wartung direkt betroffen sind wird dabei fahrzeugspezifisch festgelegt. Bei unsachgemäßem Service können unbeabsichtigt inkompatible Austauschteile oder nicht vom Fahrzeughersteller freigegebene Komponenten verbaut werden. Im Betrieb können die fehlerhaft installierten Teile zu irreparablen Schäden an anderen Systemen führen. Die derzeit einzig mögliche Schutzmaßnahme besteht in einer visuellen Prüfung. Abhilfe könnten RFID-Transponder an dem jeweiligen Verschleißteil schaffen, welche eine automatisierte Erfassung der verbauten Komponenten ermöglichen und derzeit im Bereich der Produktion und Logistik für die Automobilindustrie seit Jahren erfolgreich eingesetzt werden [7].

Das hier beschriebene Systemkonzept ermöglicht eine vereinfachte Wartung von Austauschteilen wie bspw. Filtern. Das System kann durch gespeicherte Informationen des RFIDTransponders selbständig die Komptabilität zum Fahrzeug und das Alter der zu installierenden Komponenten prüfen. Ebenso kann durch die ausgelesenen Informationen unterschieden werden, ob es sich um Original- oder Nachrüstteile handelt. Nach dem Einbau kann der Zeitpunkt des Austausches direkt in den nicht flüchtigen EEPROM-Speicher des Transponders geschrieben und diese Komponenten dadurch als gebraucht gekennzeichnet werden. Bei Ablauf der maximalen Laufzeit kann weiterhin zustandsorientiert eine Warnmeldung ausgegeben werden. So wird bei einem Service gezielt ein Austausch vorgenommen und dieser auch garantiert vom Fahrzeughalter nachvollzogen werden können.

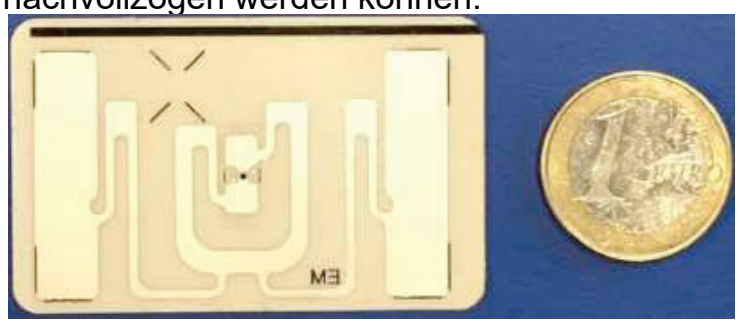

Abb. 4: UHD RFID EPC2 Transponder in der Ausführung als Klebeetikett

Bei der Auswahl von RFID-Systemen wird häufig nach dem Kriterium der Arbeitsfrequenz unterschieden. Aufgrund diverser Vorteile wurden frühzeitig Systeme im UHF-Frequenzband für die Umsetzung des Systemkonzepts ausgewählt.

Einer der technischen Hauptgründe, warum UHF-RFID (860-950 MHz) verwendet wird, ist die wesentlich höhere Lesereichweite als bei $\mathrm{HF}(13,56 \mathrm{MHz})$ und LF(125 kHz) [8]. Des Weiteren ist die Antennenabmessung grundsätz- 
lich proportional zur Wellenlänge, sodass sich bei höheren Frequenzen kleinere Antennen für die Transponder ergeben. Einschränkend ist hier zu erwähnen, dass bei induktiver Kopplung im HF- und LF- Bereich die Antennen in Spulenform ausgelegt werden können, welches im UHF-Bereich nicht realisierbar ist [8]. Im Sinne des Systemkonzepts ist die Auflösung von mehreren Transpondern mit einer Basisstation von entscheidender Bedeutung. Hierfür stellen UHF-Systeme Vielfachzugriffsverfahren bereit, welche bei niederfrequenten Systemen nur stark eingeschränkt zur Verfügung stehen. Weiterhin bieten UHF-Systeme einen deutlich höheren Datendurchsatz. Dadurch ist die Realisierung von komplexeren Kommunikationsprotokollen mit fehlererkennenden und -korrigierenden Codes möglich.

In Abb. 5 ist der typische Aufbau eines passiven UHF-RFID-Transponders zu sehen.

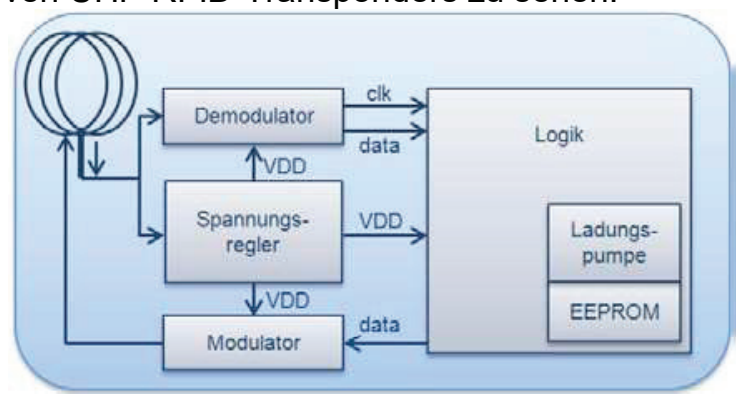

\section{Abb. 5: Aufbau eines passiven RFID-Transponders}

Durch genormte Schnittstellen können sowohl Basisstationen, als auch Transponder herstellerunabhängig kombiniert werden. Für den UHF-Bereich hat EPCglobal ein Schnittstellenprotokoll für die Luftschnittstelle definiert: Das "Class 1 Generation 2 UHF Air Interface", kurz "Gen 2". Dieses Protokoll definiert als Standardprotokoll die physikalischen und logischen Anforderungen für ein Übertragungsverfahren zwischen einem passiven RFID-Transponder und dem RFID-Lesegerät im UHF-Bereich zwischen $860 \mathrm{MHz}$ und $960 \mathrm{MHz}$. Die Datenübertragung erfolgt über eine Modulation des Rückstreuquerschnitts, wobei ausschließlich die Basisstation eine Kommunikation initiiert. Als Antikollisionsmechanismus wird das Sloted-ALOHA-Verfahren verwendet. Dieses gewährleistet, dass mehrere Transponder durch eine Basisstation aufgelöst und separat erfasst werden können.

\section{Passives Verfahren zur Auswertung des Füllstands anhand der Signaldämpfung}

In diesem Abschnitt wird ein Verfahren zur passiven Ermittlung des Füllstands in den Flüssigkeitsbehältern eines Kraftfahrzeugs vorgestellt. Dabei wird die Höhe des Flüssig- keitsstandes in einem Behälter mittels einer Basisstation erfasst, die ein elektromagnetisches Signal in den Behälter aussendet. Das ausgestrahlte Signal wird durch einen Transponder, welcher durch seine geringe Dichte auf der Oberfläche des Fluides schwimmt, detektiert und mithilfe einer Auswerteeinheit erfasst. Auf diese Weise wird eine Bestimmung des Abstands zwischen Basis und Transponder ermöglicht, welche mit der Füllhöhe des Behälters korreliert [6].

Bei diesem Verfahren handelt es sich um eine vollständig kontaktlose Messwertaufnahme, sodass der Transponder mit einer geeigneten Polymerschicht ummantelt werden kann. Da der Transponder direkt in dem zu erfassenden Medium positioniert wird, bietet der Kunststoffmantel eine elektrische, sowie eine chemische Isolation gegenüber den z.T. aggressiven Flüssigkeiten im Fahrzeug. Desweiteren kann die Kunststoffschicht als mechanischer Stoßdämpfer fungieren, da der Transponder im Fahrbetrieb der Dynamik des Fahrzeugs ausgesetzt ist.

Die Auswerteeinheit ermittelt aus der Signalstärke des empfangenen Signals die Dämpfung des ausgestrahlten Signals und kann dadurch den Abstand zum Transponder bestimmen. Das störbehaftete Arbeitsumfeld im Motorraum des Fahrzeugs mit metallischen Oberflächen und EMV-ausstrahlenden Komponenten erschweren eine Auswertung mit hoher Auflösung. Aus diesem Grund wird ein Referenz-Transponder am Boden des Behälters platziert und ermöglicht somit eine Kalibrierung des Systems. Desweiteren wird durch die Verwendung eines Referenz-Transponders eine Kompensation von alterungsbedingten Umgebungseffekten, wie z.B. Verschmutzung des Behälters, ermöglicht.

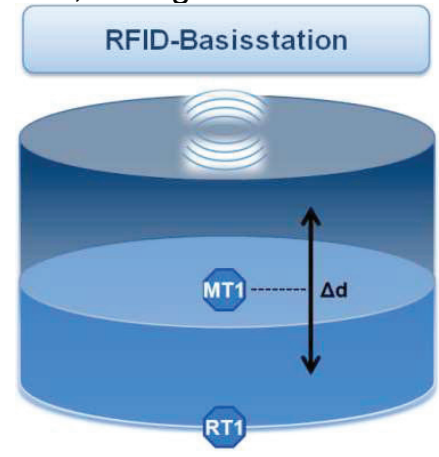

\section{Abb. 6: Behälter mit schwimmendem Mess- und Referenztransponder}

Der theoretische Freiraumdämpfungsfaktor FSPL im Vakuum wird bestimmt durch das Verhältnis der Frequenz $f$, der Lichtgeschwindigkeit $c$ und dem Radius $r$ über folgende Formel [8]: 
$F S P L(d B)=10 \cdot \log _{10}\left(\frac{4 \cdot \pi \cdot r \cdot f}{c}\right)^{2}$

Mit heutiger Low-Power-Halbleitertechnologie lassen sich integrierte Schaltkreise als RFIDTransponder mit einer Leistungsaufnahme von weniger als $20 \mu \mathrm{W}$ realisieren [9]. Der Wirkungsgrad eines integrierten Gleichrichters kann im UHF-Bereich mit 35\% angenommen werden [10]. Daraus folgend ergibt sich zum Betrieb des passiven Transponders eine Empfangsleistung von ca. $60 \mu \mathrm{W}$ an der Empfangsantenne. Dies bedeutet, dass bei einer Strahlungsleistung des Lesegerätes von 0,5 W EIRP die Freiraumdämpfung einen Wert von 39,2 dB nicht überschreiten darf, um an der Empfangsantenne noch eine ausreichende Leistung zum Betrieb des Transponders zu erhalten. Bei einer Sendefrequenz von $868 \mathrm{MHz}$ ergibt sich daraus eine maximale Reichweite von ca. $2,5 \mathrm{~m}$.

Neben der maximalen Reichweite ist für eine Bestimmung der Entfernung und damit des Flüssigkeitsfüllstandes vor allem die Auflösung der Signalauswertung von Bedeutung. Messungen mit dem AS3993 UHF Developmentkit von Austrian Microsystems haben gezeigt, dass eine Auflösung des Abstands zwischen Basis und eines passiven RFID-Transponders über die Signaldämpfung im cm-Bereich möglich ist. Bei einem Scheibenwischwassertank mit einer Tiefe von $20 \mathrm{~cm}$ konnte der Füllstand unter Laborbedingungen auf $5 \mathrm{~cm}$ genau aufgelöst werden, was bei symmetrischer Geometrie somit etwa einem Viertel des Volumens entspricht und eine ausreichende Genauigkeit für eine Komfortfunktion darstellt.

Tab. 1: Gemessene Leistungspegel zur Ermittlung der Füllstandshöhe des Fluides

\begin{tabular}{|c|c|}
\hline Abstand $\Delta d$ in $\mathrm{cm}$ & $\begin{array}{c}\text { Leistungspegel in } \\
\mathrm{dBm}\end{array}$ \\
\hline 48 & -55 \\
\hline 43 & -52 \\
\hline 38 & -50 \\
\hline 33 & -45 \\
\hline 28 & -38 \\
\hline
\end{tabular}

Voraussetzung für eine zuverlässige Detektion der Füllhöhe ist allerdings die Berücksichtigung der Richtcharakteristika der FernfeldFlächenantenne der Basisstation. Bei Bewegungen des Transponders parallel zur Ebene der Antenne ergeben sich deutliche Schwankungen im Signalpegel, welche eine Bestimmung des Füllstands verhindern. Weiterhin ist die Richtcharakteristik der TransponderAntenne ein entscheidender Faktor, da der gemessene Signalpegel stark von der Ausrich- tung des Transponders abhängt. Bei einer Drehung des Transponders parallel zur Basisantenne von 45 Grad gegenüber der optimalen Ausrichtung sinkt der gemessene Pegel bei der durchgeführten Messreihe um $10 \mathrm{dBm}$.

Eine Lösung der Problematik könnte eine Reduzierung der Freiheitsgrade in der Bewegung des Transponders durch Anpassung der Tankgeometrie darstellen. Durch Erweiterung der Konstruktion kann eine Führung für den Schwimmer mit inkludiertem Transponder in den Tank eingebracht werden, die ausschließlich eine Bewegung in eine Dimension mit Änderung des Füllstands erlaubt und somit eine zuverlässige Füllstandserfassung ermöglicht.

\section{Unterstützung der drahtlosen Energieüber- tragung bei leistungsintensiven Verbrau- chern}

Für viele Anwendungen aus dem Bereich LowPower-Sensorik ist eine Übertragung der notwendigen Energie im elektromagentischen Feld ausreichend, um die sensorische Funktion durchführen zu können. Diese Sensorsysteme haben bei aktiver Messdatenerfassung häufig eine Leistungsaufnahme im $\mathrm{mW}$ Bereich. Die Strahlungsleistung bei UHF-RFID im Frequenzband von $868 \mathrm{MHz}$ ist auf $2 \mathrm{~W}$ Effektive Strahlungsleistung (ERP) begrenzt [11]. Bei Vernachlässigung der Freiraumdämpfung ergibt sich dadurch eine maximal übertragene Leistung von 2 Watt. Dementsprechend darf ein Sensorsystem für eine zuverlässige Funktion zu keinem Zeitpunkt eine höhere Leistungsaufnahme aufweisen. Für viele der konventionellen Sensoren im Fahrzeug wäre eine solche Versorgung nicht ausreichend. Um diese Sensorsysteme dennoch betreiben zu können, kann für leistungsintensive Verbraucher eine Unterstützung der UHF-RFID-Energieübertragung durch eine weitere induktiv gekoppelte Antenne erfolgen.

Die theoretische Grundlage für die induktive Kopplung ergibt sich aus dem Gesetz von BiotSavart:

$$
H=I / 4 \pi \int_{s}(d s \times R) / r^{3}[12]
$$

Für den Verlauf der magnetischen Feldstärke entlang der Spulenachse einer runden Leiterschleife, wie sie auch als Sendeantenne in induktiv gekoppelten RFID-Systemen verwendet wird, gilt zwischen dem Spulenradius $R$, dem Spulenstrom I, der Windungszahl $\mathrm{N}$ und dem Abstand $d$ in $y$-Richtung folgende Beziehung:

$$
H=I \cdot N \cdot R^{2} / 2 \cdot \sqrt{\left(R^{2}+d^{2}\right)^{3}}
$$


Aus dieser Beziehung ist ersichtlich, dass im Gegensatz zu elektromagnetisch gekoppelten RFID-Systemen im Fernfeld mit einer Dämpfung $20 \mathrm{~dB} /$ Dekade für die induktive Kopplung im Nahfeld eine Dämpfung von 60dB/Dekade angenommen werden muss. Dementsprechend ist die Reichweite dieser Systeme mit einem akzeptablen Wirkungsgrad stark eingeschränkt.

Die Ausrichtung der Sende- und Empfängerspulen ist bei induktiver Kopplung von entscheidender Bedeutung, da diese den Wirkungsgrad einer möglichst verlustfreien Energieübertragung bestimmt. Bei der Positionierung kann grundsätzlich zwischen lateralen, verkanteten und vertikalen Fehlausrichtungen unterschieden werden, welche Verluste bei der Energieübertragung verursachen.
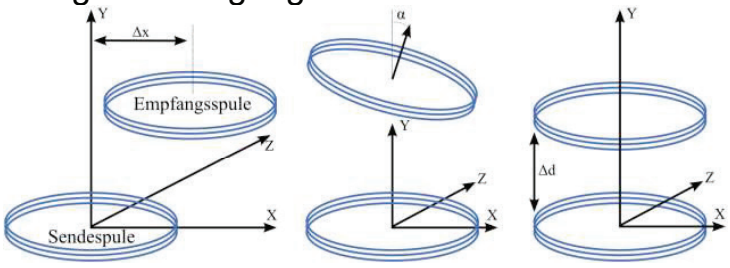

Abb. 7: Fehlausrichtung von Sende- und Empfangsspulen

Neben einem minimalen Abstand in y-Richtung hängt die Kopplung der Spulen von der maximalen wirksamen Fläche der Empfängerspule im magnetischen Feld der Sendespule ab. Ist die Empfängerspule parallel über der Sendespule zentriert und der Abstand $\Delta \mathrm{d}$ möglichst gering, so entsteht ein idealer Kopplungsfaktor von 1. Der Kopplungsfaktor kann mit folgender Gleichung ermittelt werden:

$k=\sqrt{k_{1} \cdot k_{2}}=\frac{M}{\sqrt{L_{1} \cdot L_{2}}}$

Dabei stellen $L_{1}$ und $L_{2}$ die Selbstinduktivitäten und $\mathrm{M}$ die Gegeninduktivität zwischen den beiden Spulen dar. Für die Effizienz der Energieübertragung sollten Spulen mit hoher Güte verwendet werden. Die Güte der Spulen wird definiert als Quotient des Betrags zwischen Blind- und Wirkwiderstand:

$Q=\frac{\left|X_{L}\right|}{\left|R_{L}\right|}=\frac{\omega L}{R_{L}}$

Der Übertragungsfaktor für die drahtlose Energieübertragung wird durch das Verhältnis von eingebrachter Energie an der Basisstation zu übertragener am Sensorsystem bestimmt. Zur Minimierung der Übertragungsverluste ist der magnetische Feldverlauf entscheidend. Durch eine Ferritschirmung unterhalb der Sendeantenne kann das magnetische Feld gezielt auf den Raum zwischen der Sende- und Empfängerspule begrenzt und eine ungewünschte
Abstrahlung in die Umgebung vermieden werden [13].

Die Technologie der drahtlosen Energieübertragung wird derzeit stark vom Smartphoneund Tablet-Markt getrieben und gewinnt immer mehr an Bedeutung. Für das kabellose Aufladen der mobilen Geräte haben sich inzwischen Standards wie z.B. der "Qi Standard" des Wireless Power Consortiums oder „Rezence” der Alliance for Wireless Power etabliert, die die Voraussetzung für das herstellerunabhängige Laden schaffen $[14,15]$. Zukünftig werden diese etablierten Standards eine hohe Anzahl an Produkten hervorbringen, sodass die Systeme zur drahtlosen Energieübertragung deutlich kostengünstiger werden. Dadurch könnte diese Technologie auch in einem sehr kostensensitiven Markt wie in der Automobilbranche Anwendung finden.

$\mathrm{Zu}$ Demonstrationszwecken wurde ein Muster eines energieautarken drahtlosen Sensorsystems aufgebaut, wie in Abbildung 8 dargestellt. Die zentralen Komponenten dieses Sensorknotens sind der MSP430-Low-PowerMikrocontroller und das CC2500 Funkmodul von Texas Instruments.

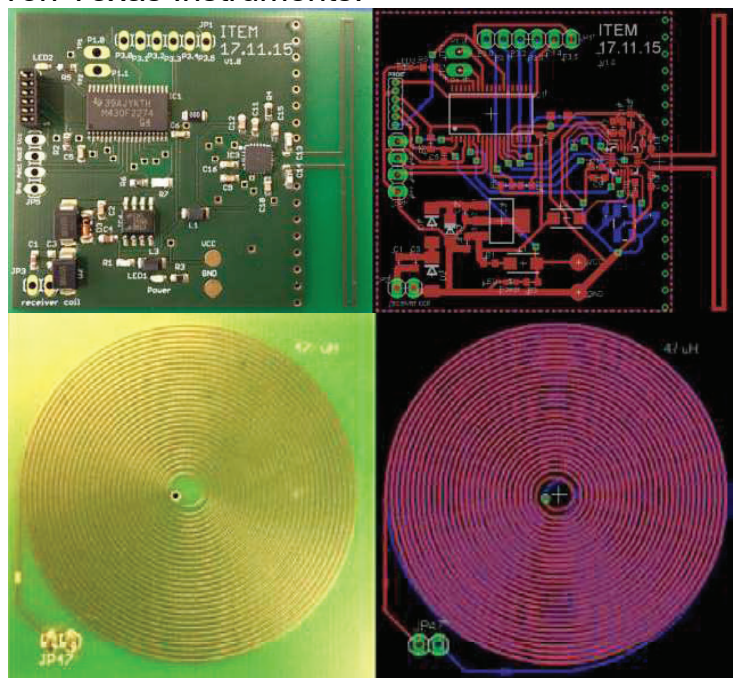

Abb. 8: Low-Power-Sensorsystem mit MSP430 Mikrocontroller und CC2500 Funkmodul (oben) und PCB-Spulen zur drahtlosen Energieübertragung (unten)

In der Abbildung 8 (unten) ist die Empfängerspule auf PCB-Basis dargestellt. Diese hat den Radius $2,3 \mathrm{~cm}$ bei einer Leiterbahnbreite von $400 \mu \mathrm{m}$, einem Leiterbahnabstand von $300 \mu \mathrm{m}$ und einer Kupferdicke von $35 \mu \mathrm{m}$. Bei einer Messung wies die Empfängerspule einen ohmschen Widerstand $R_{L}$ von $5,6 \Omega$, die Induktivität $L$ von $59 \mu \mathrm{H}$ und eine Güte $Q$ von 6,62 bei $100 \mathrm{kHz}$ auf. Als Basisboard wurde ein DC1968A Basic Transmitter Board von Linear Technologies verwendet. In einer Messreihe 
wurde der Dämpfungsfaktor bei Versatz zwischen der Empfänger- und der Sendespule ermittelt. Die Ergebnisse der Untersuchung sind in Tabelle 2 dargestellt.

Tab. 2: Drahtlos übertragene Leistung in Abhängigkeit von der Ausrichtung

\begin{tabular}{|c|c|c|c|}
\hline \multicolumn{2}{|c|}{ Ausrichtung } & \multirow{2}{*}{$\begin{array}{c}\begin{array}{c}\text { Übertragene } \\
\text { Leistung } \\
\text { in Watt }\end{array} \\
9,5 \\
\end{array}$} & \multirow{2}{*}{$\begin{array}{c}\begin{array}{c}\text { Leistungs- } \\
\text { dämpfung } \\
\text { in } \mathrm{dB}\end{array} \\
0 \\
\end{array}$} \\
\hline \multirow{5}{*}{ 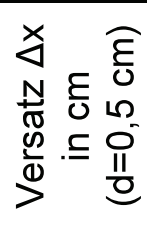 } & 0 & & \\
\hline & 0,5 & 6,98 & 1,34 \\
\hline & 1 & 3,5 & 4,34 \\
\hline & 1,5 & 0,66 & 11,58 \\
\hline & 2 & 0,25 & 15,8 \\
\hline \multirow{5}{*}{ 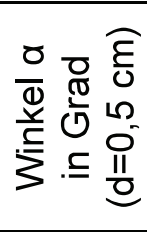 } & 10 & 5,45 & 2,41 \\
\hline & 20 & 2,8 & 5,31 \\
\hline & 40 & 0,59 & 12,07 \\
\hline & 60 & 0,28 & 15,03 \\
\hline & 90 & 0,1 & 19,78 \\
\hline \multirow{5}{*}{ 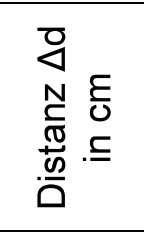 } & 0,2 & 22,41 & 0 \\
\hline & 0,5 & 9,5 & 3,73 \\
\hline & 1 & 4,47 & 7 \\
\hline & 1,5 & 1,14 & 12,94 \\
\hline & 2 & 0,65 & 15,38 \\
\hline
\end{tabular}

\section{Ausblick}

Das im Rahmen dieser Veröffentlichung vorgestellte System erweitert bestehende Entwicklungen im Bereich der energieautarken drahtlosen Sensorsysteme um das Anwendungsfeld des Kraftfahrzeugs. Zukünftig ist die Entwicklung eines aktiven kapazitiven Sensorsystems zur kontinuierlichen Füllstands- und Medienerfassung vorgesehen. Diese Funktion wird durch eine kapazitive Elektrodenstruktur in Form eines planaren, folienbasierten Messwertaufnehmers realisiert, der gegebenenfalls im Produktionsprozess direkt in den Behälter integriert oder als Nachrüstlösung an der Behälterwand appliziert werden kann. Zur Kostenreduktion ist angedacht, Elektrodenstrukturen mit elektrisch leitfähiger Tinte auf einem Polymersubstrat aufzudrucken. Um eine hohe Miniaturisierung und Energieeffizienz des Systems zu ermöglichen, ist sowohl die Elektronik zur Aufnahme und Auswertung der Messdaten, als auch ein RFID-Sendeempfänger inklusive einer geeigneten Antennenstruktur auf einem gemeinsamen folienbasierten Träger zu integrieren. Damit kann das gesamte Sensorsystem als ein kontaktloses, in sich geschlossenes planares System realisiert werden.

\section{Literaturverzeichnis}

[1] Volkswagen AG, „Selbststudienprogramm 272, Der Phaeton - Bordnetz,“ 2002.

[2] Hahn-Schickard-Gesellschaft, „Intelligente Energieautarke Kupplungen für fluidische Systeme," HSG Abschlussbericht, 2010.

[3] ZF Friedrichshafen AG, „cherryswitches.com,“ September 2012. [Online]. Available: http://www.cherryswitches.com/de/energyharvesting-technologie/. [Zugriff am 1507 2015].

[4] S. Roundy, „Energy harvesting for tire pressure monitoring system," MEMS 2008, Japan, 2008.

[5] J. Happel, J. Harmstorf, A. Kneifel und K.-L. Krieger, „Kinetic Energy Harvesting in Automotive Applications," Sensor und Test, Nürnberg, 2015.

[6] W. Benecke et al., „Verfahren und Vorrichtung zum Bestimmen des Füllstandes eines Reservoirs". Patent DE102005044188A1, 5 Mai 2007.

[7] J. Szrata, „RFID ist in der Automotive-Industrie nicht mehr aufzuhalten," RFID im Blick, Juni 2015.

[8] K. Finkenzeller, RFID Handbuch, Carl Hanser Verlag, 2015.

[9] U. Karthaus und M. Fischer, „Fully integrated passive UHF RFID transponder IC with 16.7- $\mu \mathrm{W}$ minimum RF input power," IEEE Journal of Solid-State Circuits Volume:38, p. DOI:10.1109/JSSC.2003.817249, Oktober 2003.

[10] H. Nakamoto, et al., „A Passive UHF RFID Tag LSI with $36.6 \%$ Efficiency CMOS-Only Rectifier and Current-Mode Demodulator in 0.35/spl mu/m FeRAM Technology," SolidState Circuits Conference, DOI: 10.1109/ISSCC.2006.1696166, Februar 2006.

[11] Bundesnetzagentur, ,RFID, das kontaktlose Informationssystem," [Online]. Available: http://emf2.bundesnetzagentur.de/pdf/RFID -BNetzA.pdf. [Zugriff am 26 Februar 2016].

[12] R. Paul und S. Paul, Arbeitsbuch zur Elektrotechnik, München: Springer Verlag, 1995.

[13] J. Hantschel, „Kabellose Energieübertragung: Verlustarme Spulen," Elektronik, 8 September 2015.

[14] „http://www.wirelesspowerconsortium.com,“ [Online]. Available: Qi WPC Spec 1.2. [Zugriff am 26 Februar 2016].

[15] A. Alliance, „http://airfuel.org/,“ [Online]. [Zugriff am 26 Februar 2016]. 